\title{
A Generalização do Comportamento e a Orientação dos Pais na Teoria Analitico-Comportamental
}

\author{
The Generalization of Behavior and the Orientation of Parents in \\ Analytical-Behavioral Theory
}

\author{
Cintia Figueiredo de Norões Brito*, Ana Rodrigues Costa* \\ *Universidade Fernando Pessoa,
}

\begin{abstract}
Resumo
O objetivo deste trabalho é identificar se há relação entre a ocorrência da orientação de pais/cuidadores principais e o processo de generalização. Os participantes são 38 pais/cuidadores principais que estiveram aguardando seus filhos serem atendidos em consultórios (amostra por conveniência). $\mathrm{O}$ material utilizado foi um inquérito por questionário. Verificou-se, ao analisar os resultados, que existe uma correlação positiva entre o processo de generalização e Ocorrência da orientação de pais. Assim, para promover a generalização, a orientação de pais deve ser considerada como uma intervenção frequente na terapia infantil. Em termos de conclusão, propomos algumas estratégias para efetivar a orientação de pais.

Palavras-chave: generalização; orientação de pais; terapia analitico-comportamental.
\end{abstract}

\section{Abstract}

The objective of this study is to identify if there is a relationship between the occurrence of parent / caregiver orientation and the generalization process. Participants are 38 parent / caregivers who have been waiting for their children to be seen in their practices (sample for convenience). The material used was a questionnaire survey. It was verified, when analyzing the results, that there is a positive correlation between the process of generalization and Occurrence of the parents' orientation. Thus, to promote generalization, parental guidance should be considered as a frequent intervention in child therapy. In terms of conclusion, we propose some strategies to effect parenting.

Keywords: generalization; Parent guidance; Analytical-behavioral therapy.

$\mathrm{O}$ atendimento a crianças envolve não somente o trabalho com estas mas também com outras pessoas que fazem parte da rotina do cliente como pais, irmãos, babás, avós, professores e outros profissionais que fazem parte da equipe de saúde e educação (Conte \& Regra, 2000; Moura \& Venturelli, 2004; Queiroz \& Guilhardi, 2002).

O terapeuta analítico-comportamental precisa compreender o comportamento da criança e, para isso, muitas vezes, precisa coletar informações com pessoas significativas (Conte \& Regra, 2000; Regra, 2000). Ao elaborar intervenções, necessita orientar as pessoas que estarão em contato com a criança e criar contingências que facilitem a generalização para o ambiente natural do cliente.

A intervenção com pais justifica-se a partir das seguintes premissas: a) o comportamento da criança é resultado da interseção do organismo infantil com variáveis históricas e ambientais; b) sendo mantido pelas contingências ambientais familiares; c) por estar mais tempo com as crianças e serem seus responsáveis, são os pais que dispõem dos reforçadores envolvidos, sendo eles que têm condições de alterar as contingências que controlam os comportamentos (Silvares \& Marinho, 1998). As autoras acrescentam que a função dos pais como mediadores neste processo auxilia na generalização dos ganhos terapêuticos.

É importante que o psicólogo organize contingências que facilitem a emissão do comportamento modelado em terapia para outros ambientes, promovendo a generalização (Gadelha \& Vasconcelos, 2005).

Assim, ao elaborar intervenções, o psicólogo precisa orientar as pessoas que estarão em contato com a criança e criar contingências que facilitem a generalização para o ambiente natural do cliente, ou seja, fazer a Orientação aos cuidadores da criança. Os pais/cuidadores devem, por meio do processo terapêutico, tornar-se analistas do comportamento de seus filhos e deles próprios ao invés de simples mediadores. Acredita-se que esta deve ser uma postura desenvolvida e mantida não só pelos pais, mas pelos indivíduos significativos no cotidiano da criança. Assim o processo de treinamento de pais e professores envolve um processo voltado para a análise funcional dos comportamentos emitidos pela criança e por outros membros da família.

Embora o trabalho com pais na terapia infantil tenha se mostrado efetivo em muitas situações, autores (Griest \& Forehand, 1983; Batista \& Weber, 2014; Marinho \& Silvares, 2000; Prebianchi, 2011) têm apontado dificuldades em relação aos resultados da orientação parental. "Enquanto muitos estudos de treinamentos de pais têm apontado sucesso em modificar as interações

Correspondência: Cintia de Brito, cintiafigueiredo@hotmail.com

Selección y peer-review bajo responsabilidad del Comité Organizador del XIV Congreso Internacional Galego-Portugués de Psicopedagogía 
pais-filhos, este não tem sido um caso comum. Além disso, mudanças que têm ocorrido frequentemente não têm sido duradouras" (Griest \& Forehand, 1983, p.73). Os modelos teóricos de intervenção de pais apresentados pela literatura são inquestionáveis, mas a experiência clínica e os dados empíricos têm apresentado problemas inesperados (Silvares \& Marinho, 1998). Ressaltam que muitos pais têm dificuldades de cooperar com as terapias individuais de seus filhos ou dificuldades em aplicar os procedimentos ensinados pelo treinamento de pais. Concluem que, atualmente, para se escolher qual intervenção será utilizada na abordagem de um problema comportamental infantil, precisam ser investigadas as características de pais e familiares.

O presente estudo tem como principais objetivos: a) identificar se a orientação de pais está sendo realizada pelos psicólogos que atendem crianças, com que frequência e identificar se há relação entre a ocorrência da orientação de pais/cuidadores principais e o processo de generalização.

\section{Método}

Participantes. Os participantes são pais que estiveram aguardando seus filhos serem atendidos em consultórios por psicólogos com orientação teórica analítico-comportamental infantil na cidade de Fortaleza, Ceará, Brasil. Trata-se assim de uma amostra por conveniência (Hill \& Hill, 2000).

Os questionários foram respondidos por mães $(68,4 \%)$, pais $(23,7 \%)$, pai e mãe $(5,3 \%)$ e uma avó $(2,6 \%)$ de clientes atendidos na cidade de Fortaleza/CE. Estes se declararam casados $(68,4 \%)$, solteiros $(7,9 \%)$, em união estável $(2,6 \%)$, divorciados $(15,8 \%)$ ou viúvos $(5,3 \%)$. Quanto à escolaridade, a maioria, tanto dos pais $(65,8 \%)$, quanto das mães $(78,9 \%)$, indicou possuir ensino superior completo. As crianças atendidas distribuíram-se equitativamente em função do sexo e apresentaram idades entre 2 e 15 anos $(M=7,7 ; D P=$ $3,11) ; 52,6 \%$ têm pais que fizeram ou fazem terapia (individual ou familiar).

Materiais. $\mathrm{O}$ inquérito por questionário foi elaborado com base na literatura especializada na área, sendo as perguntas formuladas a partir de resultados de pesquisas que abordam o assunto em estudo, com o objetivo de identificar se há relação entre a ocorrência da orientação de pais/cuidadores principais e o processo de generalização em situação de atendimento na clínica analítico-comportamental. É constituído por 20 itens, que devem ser respondidos através de uma escala de likert

Antes de iniciar a coleta dos dados, o instrumento foi testado por meio de dois procedimentos: análise por 4 especialistas e pré-teste com 5 sujeitos com carateristicas semelhantes às dos participantes, sendo sugeridas as modificações necessárias. Esta etapa, em que se efetuou a reflexão falada, foi responsável por mudanças no questionário de forma a viabilizar que o objetivo da pesquisa fosse alcançado.

Agrupamos os itens do questionário em categorias de forma a viabilizar a análise de correlações entre as variáveis em estudo. Este procedimento teve como embasamento a literatura estudada e permitiu a criação de categorias sendo que neste trabalho analisamos as duas que se seguem:

Ocorrência da orientação correspondente às questões 4) Você recebe orientações do(a) psicólogo sobre como agir na educação do seu(sua) filho(a) em casa ou em outros ambientes? e 5) Qual a frequência das orientações? O objetivo desta categoria é identificar se a orientação de pais está acontecendo e, em caso afirmativo, com qual frequência. Em outras palavras, é necessário verificar se os psicólogos estão realizando a orientação parental e com quanto tempo de intervalo, levando-se em consideração a opinião dos pais.

Ocorrência da generalização - Percepção dos pais (Questão 9) Você percebe resultados satisfatórios no comportamento do seu(sua) filho(a) depois de seguir as orientações? Esta categoria envolve uma pergunta referente à percepção dos pais sobre a ocorrência da generalização. A questão investiga se eles identificam resultados satisfatórios no comportamento dos filhos após seguirem as orientações do psicólogo. Respostas positivas indicam que os pais percebem a ocorrência da generalização no tratamento das crianças.

Procedimento. A partir do estudo da referência bibliográfica, foi elaborado pela pesquisadora e pela orientadora, o inquérito por questionário que serviu para recolher dados que permitissem identificar se há relação entre a ocorrência da orientação de pais/cuidadores principais e o processo de generalização em situação de atendimento na clínica analítico-comportamental infantil.

Elaboramos um projeto de pesquisa que foi submetido à Comissão de Ética da Universidade Fernando Pessoa que teve parecer positivo.

Realizamos uma revisão de literatura especializada na área, envolvendo autores nacionais e internacionais, de forma a que o tema da generalização de ganhos terapêuticos no contexto de orientação de pais fosse compreendido em sua abrangência.

A preparação para a execução da pesquisa envolveu o contato com professores da Universidade Federal do Ceará para a indicação de alunos do curso de psicologia que tivessem interesse em participar da fase de aplicação dos questionários. A pesquisadora selecionou quatro alunos por meio de entrevistas e os treinou para aplicar os questionários.

Para selecionar os locais onde decorreria a pesquisa, a investigadora entrou em contato com psicólogos analítico-comportamentais de Fortaleza, explicou os procedimentos, objetivos e documentos do estudo, solicitando a permissão para aplicação dos questionários. Os profissionais e as clínicas foram informados oralmente pela pesquisadora e consentiram com a investigação.

Os alunos de Psicologia iniciaram a aplicação individual dos questionários aos pais de pacientes que estavam aguardando, na sala de espera, seus filhos serem atendidos por psicólogos analítico-comportamentais. Esta fase envolveu a apresentação de uma folha de rosto contendo informações sobre a pesquisa e o consentimento informado para assinatura dos participantes antes de 
responder o questionário. Após a assinatura do mesmo e dos esclarecimentos considerados necessários, era entregue o questionário que, depois de respondido, deveria ser depositado em uma urna para garantir o caráter anônimo do procedimento.

Foram aplicados 38 questionários em pais de pacientes de 8 psicólogos que se declararam analítico-comportamentais da cidade de Fortaleza. Depois de recolhidos os dados estes foram trabalhados recorrendo ao Statistical Package for the Social Sciences (SPSS).

\section{Resultados}

Para avaliar a consistência interna calculamos o Alfa de Cronbach, considerando o agrupamento dos itens. O resultado dessa análise revelou um alfa de 0,71 , que, segundo a literatura, pode ser compreendido como satisfatório (Nunnally, 1978).

Os dados coletados foram analisados a partir do SPSS (Versão 22). Além das estatísticas descritivas (Tendência Central e Dispersão), realizaram-se análises de correlação (Spearman) a fim de observar as relações entre as categorias estabelecidas.

Com o objetivo de identificar se a orientação de pais está sendo realizada pelos psicólogos, o item 4 avaliou que $78,9 \%$ dos pais considera que recebem, com uma frequência entre "Sempre" e "Muitas vezes", orientações sobre como agir na educação do seu(sua) filho(a) em casa ou em outros ambientes. O percentual de $18,4 \%$ foi verificado para "Às vezes" e de $2,6 \%$, para "Raramente".

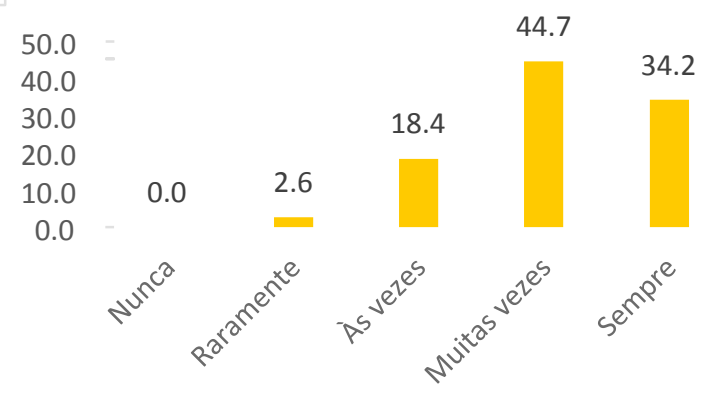

Figura 1. Distribuição das respostas para o item 4.

Além de identificar, no item anterior, que as orientações estão sendo feitas, é necessário verificar a frequência com que acontecem. $\mathrm{O}$ item 5 avaliou que $81 \%$ dos psicólogos realizam orientações de pais pelo menos uma vez ao mês.

A distribuição das respostas no gráfico, 2,7\% "Quinzenal" em oposição a 37,8\% "Semanal" ou 40,5\% "Uma vez ao mês", possibilita sugerir que, para responder a este item, os pais consideraram diferentes modalidades de orientação, como sessões no consultório, ligações telefônicas ou mensagens.

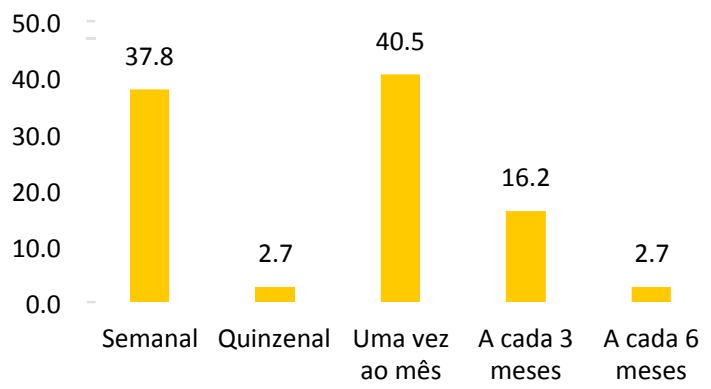

Figura 2. Distribuição das respostas para o item 5 .

Quanto ao item 9 verificou-se que $84,3 \%$ da amostra percebe ganhos terapêuticos relacionados ao seguimento das orientações com frequência entre "Sempre" e "Muitas vezes", sendo 15,8\% o percentual relacionado àqueles que identificaram a frequência "às vezes".

Este item diz respeito à constatação dos pais de que o processo de generalização ocorreu ou não, pois os resultados em ambiente de casa são, justamente, o objetivo do processo de generalização.

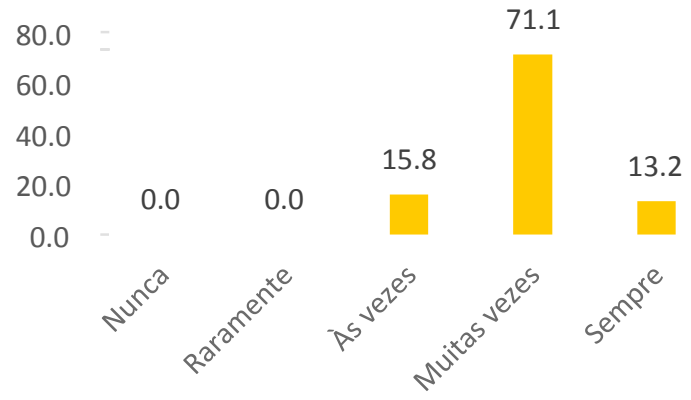

Figura 3. Distribuição das respostas para o item 9.

As categorias, definidas a partir da bibliografia na área, foram compreendidas por meio de análises de correlações (Spearman) com uso do SPSS (Versão 22).

No que tange à relação entre as categorias Ocorrência da orientação e Generalização, os resultados demonstraram uma correlação significativa, moderada e positiva $(r=0,41, p<0,05)$.

\section{Discussão}

Os resultados indicam que, na medida em que se amplia a frequência das orientações, se constata um aumento do processo de generalização (pais seguirem as orientações de pais).

Estes resultados são semelhante aos dados encontrados por Bolsoni-Silva (2007) que, ao realizar uma revisão bibiográfica com estudos que avaliaram a efetividade das intervenções com pais para lidar com os comportamentos problemas dos filhos, identificou que houve resultados satisfatórios, como: aumento das práticas parentais positivas; redução das práticas parentais negativas; redução de problemas de comportamento.

Esta análise permite alertar os psicólogos para a importância da realização da orientação de pais para a obtenção de melhores resultados terapêuticos, principalmente em relação ao processo de generalização. A existência de uma correlação positiva 
significativa, e moderada entre a orientação de pais e a generalização demonstra que o psicólogo deve considerar a orientação de pais como uma intervenção frequente na terapia infantil.

A realização da presente pesquisa permitiu atingir os objetivos aos quais se propôs e elaborar conclusões sobre a relação entre a ocorrência da orientação de pais/cuidadores principais e o processo de generalização em situação de atendimento na clínica analítico-comportamental infantil.

Como contribuição para o atendimento clínico infantil, observou-se que o processo de generalização de ganhos terapêuticos adquiridos em consultório para outros ambientes pode ter melhores resultados quando ocorre a realização da orientação de pais.

Como limitações do estudo, destaca-se o número reduzido de participantes e o facto de ser uma amostra de conveniência, o que impede a generalização dos resultados. Também o uso de um instrumento baseado no relato verbal, com toda a desejabilidade social que isso implica, sem associação com a observação direta ou com a recolha de dados junto dos psicólogos.

Apesar das limitações, deve-se ter em conta que, mesmo com uma amostra considerada pequena $(N=38)$, foi possível constatar correlações significativas, positivas e moderdas entre as variáveis estudadas. Entende-se que o resultado merece destaque, uma vez que a significância nas análises de correlação pode ter seus valores inflacionados quando se amplia o tamanho da amostra.

Mais do que responder perguntas, a grande contribuição deste trabalho é o questionamento acerca do processo de orientação de pais. Acredita-se que as inquietações com as dificuldades que são encontradas na prática clínica podem ser responsáveis pelo movimento de pesquisa e de constante produção de conhecimento. As respostas não devem funcionar como ponto de estagnação do estudo científico, e sim como um ponto de partida para outras perguntas, contribuindo para o enriquecimento científico.

\section{Referências}

Batista, A. P., \& Weber, L. N. D. (2014). Análise de programas direcionados a comportamentos exteriorizados na infância e adolescência. Psicol. Argum,32(78), 157-168.

Bolsoni-Silva, A. T. (2007). Intervenção em grupo para pais: descrição de procedimento. Temas em psicologia, 15(2), 217-235.

Conte, F. C. S., \& Regra, J. A. G. (2000). A psicoterapia comportamental infantil: Novos Aspectos. Estudos de caso em psicologia clínica comportamental infantil, 1, 79-136.

Gadelha,Y. A., \& Vasconcelos, L. A. (2005). Generalização de estímulos: aspectos conceituais, metodológicos e de intervenção. J. Abreu Rodrigues \& M. R. Ribeiro, Análise do comportamento. Pesquisa, teoria e aplicação. Porto Alegre: Artmed, 139-158.

Griest, D. L., \& Forehand, R. (1983). How Can I Get Any Parent Training Done With All These Other Problems Going On? The Role of Family Variables in
Child Behavioral Therapy. Child \& Family Behavior Therapy, 4(1), 73-80.

Hill, M.M. \& Hill, A. (2000). Investigação por Questionário.Lisboa: Edições Sílabo.

Marinho, M. L., \& Silvares, E. F. M. (2000). A intervenção clínica comportamental com famílias. Estudos de caso em psicologia clínica comportamental infantil, 1, 139-174.

Moura, C. B. D., \& Venturelli, M. B. (2004). Direcionamentos para a condução do processo terapêutico comportamental com crianças. Revista Brasileira de Terapia Comportamental e Cognitiva, 6(1), 17-30.

Nunnally, J. C. (1978). Psychometric theory. New York: McGraw-Hill.

Prebianchi, H. B. (2011). Orientação de pais no processo de psicoterapia infantil de grupo. Psicologia em Revista, 17(1), 135-145.

Queiroz, P. P., Guilhardi, H. J. (2002). Redução da agressividade e hiperatividade de um menino pelo manejo direto das contingências de reforçamento: um estudo de caso conduzido de acordo com a terapia por contingências. Sobre comportamento e cognição: contribuições para a construção da teoria do comportamento, 10, 249-270.

Regra, J. A. (2000). Formas de trabalho na psicoterapia infantil: mudanças ocorridas e novas direções. Revista Brasileira de Terapia Comportamental e Cognitiva, 2(1), 79-101.

Silvares, E. F. M. \& Marinho, M. L. (1998). Ampliando la intervención psicológica a la família en la terapia conductual infantil. Psicologia Conductual, 6(3), 617-628 